\title{
Volume Measurement
}

National Cancer Institute

\section{Source}

National Cancer Institute. Volume Measurement. NCI Thesaurus. Code C74720.

The determination of the volume of a sample. 